\title{
Usefulness of Cardiovascular Magnetic Resonance Imaging in a Patient with Cardiac Involvement of Systemic Sclerosis
}

\author{
Shingo Ota ${ }^{1}$, Motoki Taniguchi ${ }^{1}$, Yosuke Katayama ${ }^{1}$, Yuichi Ozaki ${ }^{1}$, Keisuke Satogami ${ }^{1}$, \\ Yasushi Ino ${ }^{1}$, Naoki Yamamoto ${ }^{2}$, Koji Onoda ${ }^{2}$ and Atsushi Tanaka ${ }^{3}$
}

\begin{abstract}
:
A 72-year-old Japanese woman with systemic sclerosis was admitted to our hospital because of symptoms of heart failure. Cardiovascular magnetic resonance (CMR) imaging had shown that extensive myocardial fibrosis secondary to systemic sclerosis was the main cause of heart failure. One month after CMR, she had complete atrioventricular (AV) block. It was suggested that the progression of fibrosis to the AV node caused complete AV block. This case report has clinical implications in highlighting the fact that CMR is useful for not only evaluating the present pathophysiology but also predicting future adverse events in patients with systemic sclerosis.
\end{abstract}

Key words: systemic sclerosis, heart failure, cardiovascular magnetic resonance imaging, complete atrioventricular block

(Intern Med Advance Publication)

(DOI: 10.2169/internalmedicine.8418-21)

\section{Introduction}

Patients with systemic sclerosis have multiple cardiovascular manifestations, such as electrical abnormalities, valvular involvement, myocardial involvement, pericardial involvement, and vascular involvement (1); therefore, it is not easy to accurately understand the pathophysiology of heart failure in patients with systemic sclerosis. Cardiac magnetic resonance (CMR) imaging is a useful method for understanding the underlying pathophysiology of heart failure and predicting the therapeutic response and future adverse cardiac events (2).

We herein report a patient with systemic sclerosis for whom CMR imaging was useful in understanding the pathophysiology of heart failure.

\section{Case Report}

A 72-year-old Japanese woman became aware of stiffening of both fingers from October 2018, and Raynaud's phenomenon occurred from January 2020. In January 2019, she was diagnosed with limited cutaneous systemic sclerosis characterized by skin thickness of bilateral fingers and face, mild interstitial lung disease, and an anticentromere antibody level of $27.9 \mathrm{U} / \mathrm{mL}$, which is outside the reference range $(<$ 10.0 U/mL). In February 2019, she first visited our department to be screened for cardiac disorders.

An electrocardiogram revealed sinus rhythm with complete right bundle branch block (CRBBB). Echocardiography showed a transtricuspid pressure gradient (TRPG) of 12 $\mathrm{mmHg}$, with no findings suggestive of pulmonary hypertension. A slightly dilated LV with an end-diastolic dimension of $51 \mathrm{~mm}$, normal LV ejection fraction (EF) of $60 \%$, and mild aortic valve regurgitation (AR) with noncoronary cusp prolapse were observed. Since her first visit, we have repeated the electrocardiogram and echocardiogram at least once a year.

In March 2021, she was admitted to our hospital because of peripheral edema and dyspnea on mild effort, which corresponded to New York Heart Association Function Classification III. Upon presentation, her oxygen saturation on room air was $94 \%$, pulse rate was 78 beats/min with an irregular rhythm, and blood pressure was 176/88 mmHg. On auscul-

${ }^{1}$ Department of Cardiovascular Medicine, Shingu Municipal Medical Center, Japan, ${ }^{2}$ Department of Cardiovascular Surgery, Shingu Municipal Medical Center, Japan and ${ }^{3}$ Department of Cardiovascular Medicine, Wakayama Medical University, Japan Received: July 29, 2021; Accepted: October 14, 2021; Advance Publication by J-STAGE: November 27, 2021 Correspondence to Dr. Shingo Ota, wakayama_hirosaki@yahoo.co.jp 
A

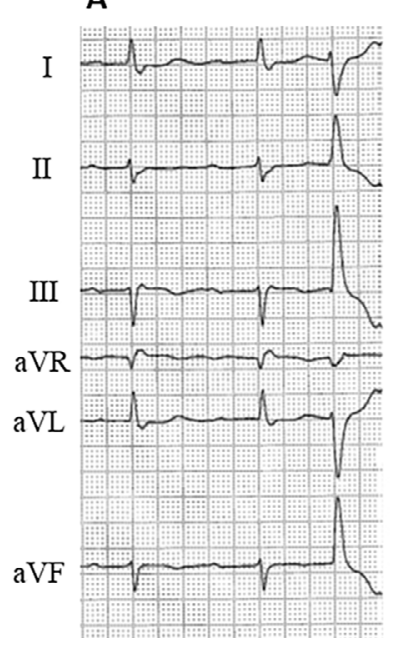

B

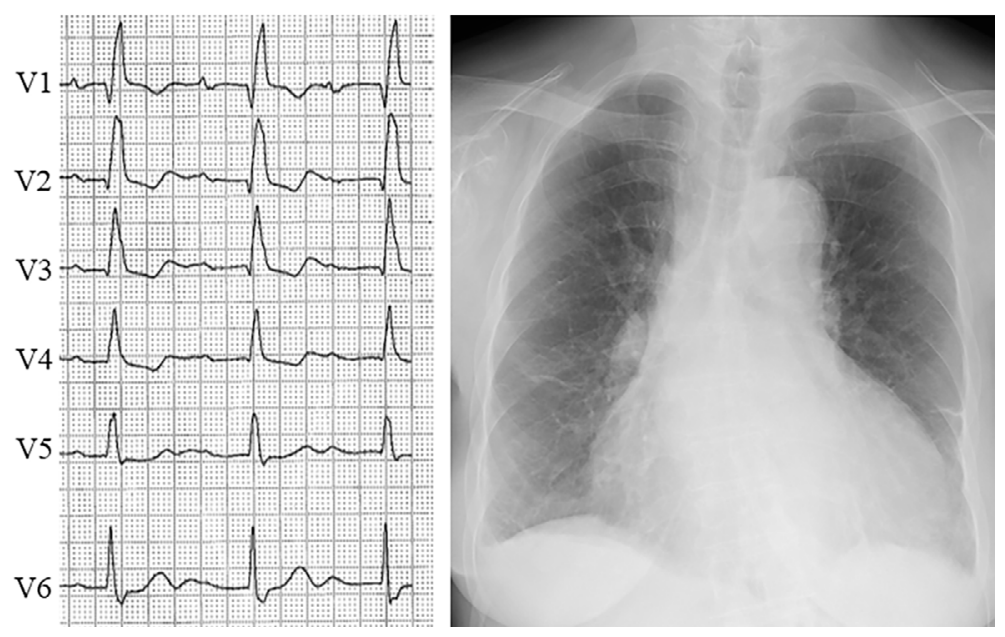

Figure 1. Baseline electrogram and chest radiography on admission. An electrocardiogram showing a Wenckebach second-degree atrioventricular block with complete right bundle branch block (A). Chest radiography revealing cardiomegaly with pulmonary congestion and slight pleural effusion (B).

tation, there was a diastolic regurgitant murmur at Erb's area and a systolic regurgitant murmur at the apex; wet rales in the lung fields were audible. Marked pretibial edema and jugular venous distension were observed. Skin thickening of the fingers of both hands extending proximal to the metacarpophalangeal joints was also observed. Blood tests revealed a brain natriuretic peptide (BNP) level of $776.3 \mathrm{pg} / \mathrm{mL}$, which is outside the reference range $(<18.4 \mathrm{pg} / \mathrm{mL})$. The estimated glomerular filtration rate was $46 \mathrm{~mL} / \mathrm{min} / 1.73 \mathrm{~m}^{2}$. An electrocardiogram showed a Wenckebach second-degree atrioventricular (AV) block with CRBBB (Fig. 1A). Chest radiography images revealed cardiomegaly with pulmonary congestion and slight pleural effusion (Fig. 1B). Echocardiography revealed a remarkably dilated LV with an enddiastolic dimension of $62 \mathrm{~mm}$, a mildly reduced LV EF of $47 \%$, and moderate functional mitral valve regurgitation due to tethering. The AR jet due to noncoronary cusp prolapse was eccentric and wide, but the pressure half time was 649 $\mathrm{ms}$, and there was no holodiastolic retrograde flow in the descending aorta, which was suggestive of mild AR. Echocardiography also showed no right ventricular dilatation and decreased wall motion, but a TRPG of $35 \mathrm{mmHg}$ suggested mild pulmonary hypertension.

CMR imaging was subsequently performed. All CMR imaging studies were performed using a 3-T cardiac-dedicated system. The CMR imaging protocol included routine cine imaging for the evaluation of the cardiac structure and function and late gadolinium enhancement (LGE) imaging for the evaluation of myocardial scarring. In addition, phasecontrast velocity-encoded imaging was performed to evaluate the regurgitation volume and fraction at the sinotubular junction. Cine imaging revealed LV dilatation and diffuse LV hypokinesis. Phase-contrast velocity-encoded imaging revealed a regurgitation volume and fraction of $18 \mathrm{~mL}$ and $25 \%$, respectively, which were suggestive of mild AR
(Fig. 2A). LGE imaging revealed diffuse subendocardial late enhancement in the basal and mid LV walls and partial late enhancement in the right ventricular wall (Fig. 2B). Although non-ischemic cardiomyopathies, including cardiac amyloidosis and cardiac sarcoidosis, were considered based on the findings of LGE imaging, we confirmed by coronary angiography that the coronary arteries were normal. The serum and urine immunofixation tests revealed no immunoglobulin monoclonal protein, and technetium-99 mpyrophosphate scintigraphy showed a grade 0 myocardial uptake. Serum soluble interleukin-2 receptor and angiotensin-1-converting enzyme levels were within the reference ranges, and bilateral hilar lymphadenopathies were not observed. Based on these findings, cardiac amyloidosis and cardiac sarcoidosis were excluded.

There was no complication with autoimmune rheumatic disease other than systemic sclerosis. Since the time of the diagnosis with systemic sclerosis, she had taken $10 \mathrm{mg}$ of prednisolone a day. We were unable to evaluate active inflammation via an endomyocardial biopsy or ${ }^{18} \mathrm{~F}$ fluorodeoxyglucose positron emission tomography; however, active myocarditis was considered unlikely because she has taken prednisolone, and there was no pericardial effusion. Eventually, she was diagnosed with cardiac involvement of systemic sclerosis.

During hospitalization, she was treated with diuretics, angiotensin II receptor blocker, and spironolactone, and the symptoms of heart failure improved. Due to concerns about worsening AV conduction, we did not prescribe a betablocker. Monitor-ECG showed first-degree AV block and Wenckebach second-degree AV block but not advanced AV block. We decided that there was no indication for a pacemaker and discharged her after a two-week hospital stay.

In April 2021, at the first visit after discharge, she did not have symptoms of heart failure or syncope; however, the 

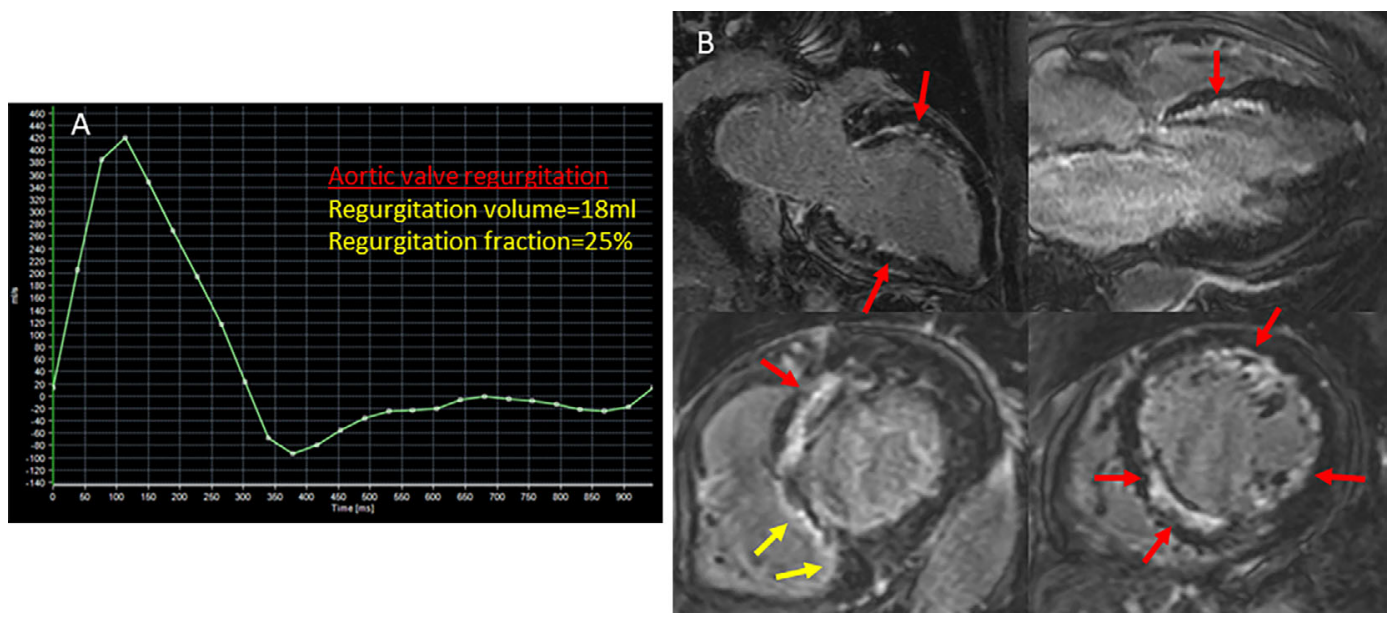

Figure 2. Cardiovascular magnetic resonance imaging. Phase-contrast velocity-encoded imaging revealed a regurgitation volume of $18 \mathrm{~mL}$ and regurgitation fraction of $25 \%$, which is suggestive of mild aortic valve regurgitation (A). LGE imaging revealed diffuse subendocardial late enhancement in the basal and mid LV walls (red arrows) and partial late enhancement in the right ventricular wall (yellow arrows) (B).

February 2019

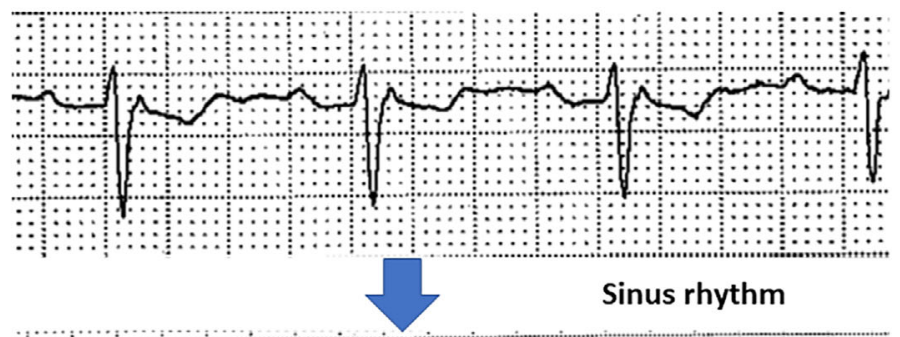

March 2021

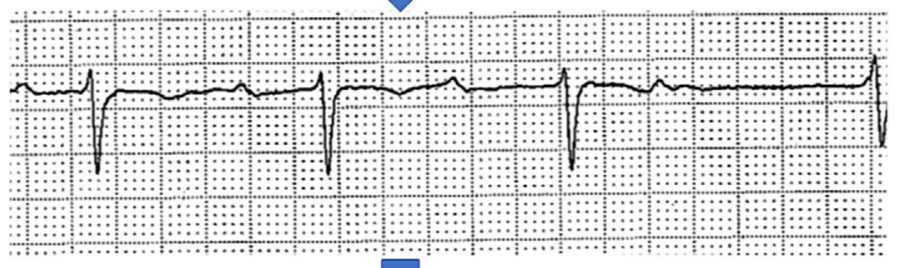

April 2021

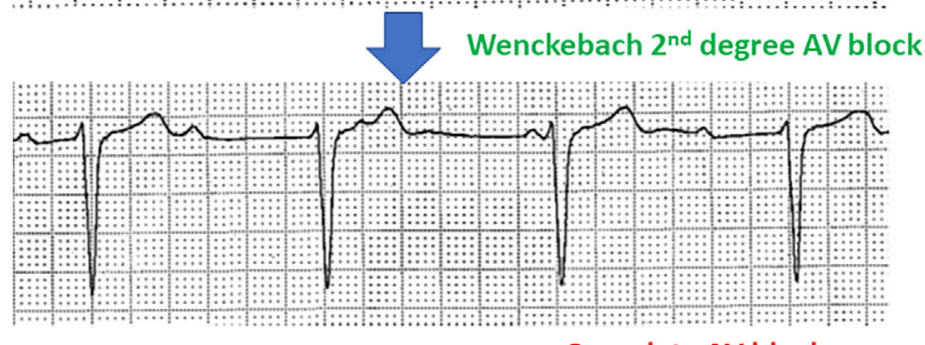

Complete AV block

Figure 3. Changes in the findings on the electrocardiogram over time. Atrioventricular (AV) conduction deteriorates over time, eventually leading to complete AV block.

ECG showed complete AV block (Fig. 3). No exacerbation of interstitial lung disease or deterioration of the renal function due to increased activity of systemic sclerosis was observed. Because of the complete AV block with heart failure, we inserted a permanent pacemaker (Fig. 4), and oral furosemide $(20 \mathrm{mg})$, tolvaptan $(3.75 \mathrm{mg})$, spironolactone $(12.5$ $\mathrm{mg})$, candesartan (8 mg), and metoprolol (2.5 mg) were administered.

\section{Discussion}

This case was an example of systemic sclerosis wherein CMR imaging showed that extensive myocardial fibrosis were the main causes of heart failure, and who had complete AV block.

LGE-CMR imaging is a useful method for determining the underlying pathophysiology of heart failure by visualizing the myocardial scar (2). Quantification of AR by CMR 


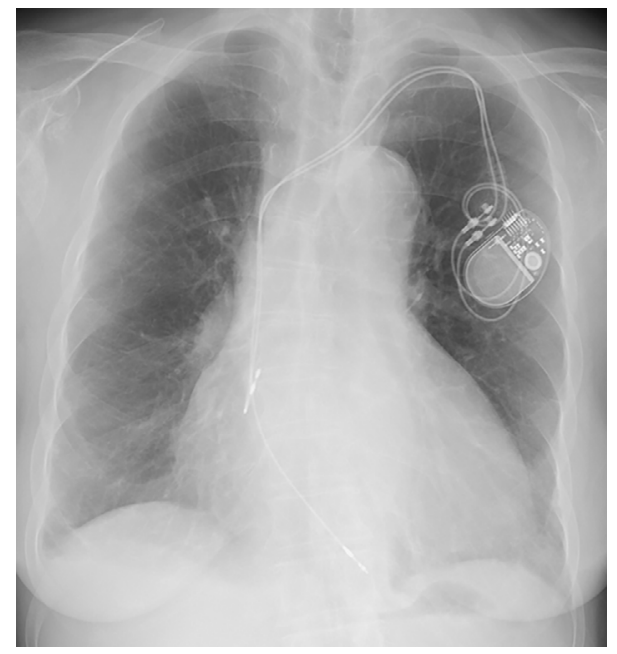

Figure 4. Chest radiography after pacemaker implantation. A permanent pacemaker was inserted because of complete atrioventricular block with heart failure.

imaging, which relies on phase-contrast velocity-encoded assessment of antegrade and retrograde flow at the sinotubular junction, is given a Class I recommendation for the assessment of AR severity (3). In the present case, CMR imaging confirmed mild AR and identified diffuse myocardial scarring due to systemic sclerosis as the underlying mechanism of heart failure. A previous study reported that there is a four-fold increased relative risk of AR in patients with systemic sclerosis (4). The pathophysiology of AR in this case was noncoronary cusp prolapse, and thickening of the aortic valve with regurgitation has been previously reported in systemic sclerosis (1). This previously reported morphological finding was not observed in the present case. Thus, the relationship between AR and systemic sclerosis was unclear in this patient.

Atrial fibrillation, flutter, or paroxysmal supraventricular tachycardia has been reported in 20\%-30\% of patients with systemic sclerosis (5). In addition, up to $67 \%$ of patients with systemic sclerosis have ventricular arrhythmias (5). Conduction disorders in systemic sclerosis are mostly due to fibrosis of the sinoatrial node. Abnormal ECG findings and bundle and fascicular blocks occur in $25 \%-75 \%$ of patients with systemic sclerosis; however, second-and third-degree $\mathrm{AV}$ blocks are rare $(<2 \%)(6)$. In the present case of systemic sclerosis, exacerbation of AV conduction, which was suggested to have been caused by progressive scarring, was documented over time. Paul et al. reported complete AV block in systemic sclerosis with characterization on CMR imaging (7). They reported that myocardial fibrosis secondary to systemic sclerosis was the underlying pathophysiology of complete AV block via CMR imaging performed at the time of pacemaker indication; however, we found CMR imaging useful for understanding the mechanisms underlying heart failure and predicting exacerbation of AV conduction disorders before a pacemaker is indicated.

Initially, we planned surgical aortic valve replacement be- cause we considered the main cause of heart failure to be AR. The pressure half time value assessed by echocardiography suggested mild AR; however, we considered the severity of AR as assessed by echocardiography to have been underestimated, as the AR jet was wide, and the LV dilatation was progressing. Since CMR imaging confirmed that the severity of $A R$ was mild and there was widespread fibrosis in the LV, we judged the main pathophysiology of heart failure to be LV remodeling due to extensive fibrosis secondary to systemic sclerosis. At the Heart Team Conference, based on the CMR imaging findings, we decided to perform careful follow-up in consideration of the possibility that AV conduction disorder would worsen with the progress of fibrosis. We were thus able to implant a pacemaker at the appropriate time. This case report has clinical implications in highlighting the fact that CMR imaging is useful for not only evaluating the present pathophysiology but also predicting future adverse events in patients with systemic sclerosis.

Sano et al. reported that the frequency of LGE was $17.5 \%$, and LGE was distributed mainly in the basal to mid inter-ventricular septum and the right ventricular insertion points but involved all myocardial regions in patients with systemic sclerosis (8). They also reported that more patients with LGE were symptomatic and had ECG abnormalities as well as a low LVEF than those without LGE. The present patient demonstrated multiple points of LGE, mainly in the LV subendocardium. She also had ECG abnormalities, such as AV conduction disorders, CRBBB, and LV dysfunction, which are compatible with the findings of the previous study.

\section{Follow-up}

We implanted a permanent pacemaker in May 2021. After the pacemaker was implanted and standard medical therapies, including a beta-blocker, were started, atrial and ventricular pacing rhythm was observed. The BNP level decreased to $191.0 \mathrm{pg} / \mathrm{mL}$, but the LV function did not improve. We feel that it may not be appropriate to evaluate the therapeutic response yet, as only about four months have passed since adequate device implantation and medical therapies were performed.

The current guideline recommends an implantable cardioverter defibrillator (ICD) or cardiac resynchronization therapy (CRT) be implanted in patients with an LVEF of $\leq$ $35 \%$ despite standard medical therapy (9). The LVEF in this patient was $47 \%$, and she had not received full standard medical therapies. Furthermore, non-sustained ventricular tachycardia was not detected in this patient; therefore, we implanted a permanent pacemaker according to the recommendation of the current guideline. Recently, we reported that multiple points of LGE were an independent predictor of adverse cardiac events in patients with non-ischemic cardiomyopathy (10). This patient had multiple points of LGE; therefore, she was considered to carry a high risk of fatal ventricular tachycardia and exacerbation of heart failure. We 
will carefully perform echocardiography and a Holter-ECG and adequately upgrade to an ICD or CRT.

The authors state that they have no Conflict of Interest (COI).

\section{Acknowledgement}

We are grateful to Katsuyuki Sakaji, RT, Shohei Miza, RT and Norifumi Kakehashi, RT for performing the CMR image acquisition.

\section{References}

1. Lambova S. Cardiac manifestations in systematic sclerosis. World J Cardiol 6: 993-995, 2014.

2. Karamitsos TD, Francis JM, Myerson S, et al. The role of cardiovascular magnetic resonance imaging in heart failure. $\mathrm{J}$ Am Coll Cardiol 54: 1407-1424, 2009.

3. Nishimura RA, Otto CM, Bonow RO, et al. 2014 AHA/ACC guidelines for the management of valvular heart disease: a report of the American College of Cardiology/American Heart Association task force on practice guidelines. J Am Coll Cardiol 63: 57185, 2014.

4. Sheraz A Butt, Jørgen L Jeppesen, Christian Torp-Pedersen, et al. Cardiovascular Manifestations of Systemic Sclerosis: A Danish
Nationwide Cohort Study. J Am Heart Assoc 8: e013405, 2019.

5. Seferović PM, Ristić AD, Maksimović R, et al. Cardiac arrhythmias and conduction disturbances in autoimmune rheumatic diseases. Rheumatology 45: iv39-iv42, 2006.

6. Janosik DL, Osborn TG, Moore TL, et al. Heart disease in systemic sclerosis. Semin Arthritis Rheum 19: 191-200, 1989.

7. Paul Guzik, Audrey Han, Samantha C Shapiro, et al. Complete Heart Block in Systemic Sclerosis with Characterization on Cardiac MRI. Methodist Debakey Cardiovasc J 16: 162-166, 2020.

8. Makoto Sano, Hiroshi Satoh, Kenichiro Suwa, et al. Characteristics and clinical relevance of late gadolinium enhancement in cardiac magnetic resonance in patients with systemic sclerosis. Heart Vessels 30: 779-788, 2015.

9. Akihiko Nogami, Takashi Kurita, Haruhiko Abe, et al. JCS/JHRS 2019 Guideline on Non-Pharmacotherapy of Cardiac Arrhythmias. Circ J 85: 1104-1244, 2021.

10. Ota S, Orii M, Nishiguchi $T$, et al. Implications of multiple late gadolinium enhancement lesions on the frequency of left ventricular reverse remodeling and prognosis in patients with non-ischemic cardiomyopathy. J Cardiovasc Magn Reson 23: 32, 2021.

The Internal Medicine is an Open Access journal distributed under the Creative Commons Attribution-NonCommercial-NoDerivatives 4.0 International License. To view the details of this license, please visit (https://creativecommons.org/licenses/ by-nc-nd/4.0/).

\section{(C) The Japanese Society of Internal Medicine} Intern Med Advance Publication 